\title{
Spatial Pattern Formation in a Catalytic Surface Reaction: The Facetting of $\mathrm{Pt}(110)$ in $\mathrm{CO}+\mathrm{O}_{2}$
}

\author{
J. Falta, R. Imbihl, ${ }^{(a)}$ and M. Henzler \\ Institut für Festkörperphysik, Universität Hannover, Appelstrasse 2, D-3000 Hannover 1, Federal Republic of Germany
}

(Received 11 December 1989)

\begin{abstract}
A detailed LEED beam profile analysis of the structural changes induced by the catalytic CO oxidation on $\mathrm{Pt}(110)$ has been carried out with a high-resolution instrument. Under conditions where the $1 \times 2 \rightleftharpoons 1 \times 1$ phase transition also takes place one observes spatial pattern formation via a facetting process. Facets of roughly uniform size and orientation build up a regular array characterized by a lateral periodicity of 70 lattice units along [1ㅣㅣ. The mechanism is discussed in terms of a nonequilibrium structure arising from kinetic instabilities.
\end{abstract}

PACS numbers: $61.14 . \mathrm{Hg}, 82.20 . \mathrm{Mj}, 82.65 . \mathrm{Jv}$

A catalytic surface reaction can modify the structure of a catalyst surface on quite different length scales ranging from local adsorbate-induced reconstructions to the formation of facets of macroscopic size. ${ }^{1}$ An example where such changes can be observed under welldefined conditions is the facetting of a $\mathrm{Pt}(110)$ surface under the influence of the catalytic $\mathrm{CO}$ oxidation. ${ }^{2,3}$ The facetting there occurs if reaction conditions are adjusted so that the $\mathrm{CO}$-induced $1 \times 2 \rightleftharpoons 1 \times 1$ phase transition (PT) also takes place. Because of the different density of surface atoms the formation and removal of the $1 \times 2$ ("missing-row") structure in the PT requires the movement of $50 \%$ of the surface atoms. ${ }^{4}$ Although it is evident that the PT can thus provide the mass transport necessary for facetting, an understanding of the process is, on the whole, still lacking. The observation that the facetting requires the presence of both gases, $\mathrm{CO}$ and $\mathrm{O}_{2}$, seem to oppose the idea of a classical thermodynamical driving force. On the other hand, the existence of kinetic oscillations in the same system under similar conditions, e.g., at higher temperature, suggests a decisive role of kinetic instabilities, in which case the facetted surface should be described as a stationary nonequilibrium structure, e.g., a structure of the Turing type. ${ }^{5}$

In this Letter we report the results of a detailed LEED beam profile analysis of the facetted $\mathrm{Pt}(110)$ surface carried out with a high-resolution instrument. The results demonstrate that the surface reaction not only leads to a preferential orientation of the facets as observed before, ${ }^{2}$ but moreover introduces a regular spacing between the facets such that a symmetric sawtoothlike array is formed by facets of uniform size and orientation. We therefore consider the facetted $\mathrm{Pt}$ (110) surface as an example for spatial pattern formation in a catalytic surface reaction similar to spatial pattern formation in other nonequilibrium systems in physics. The mechanism which is responsible for the facetting is described in terms of local structural changes initiated by local $\mathrm{CO}$ coverage fluctuations via the $\mathrm{CO}$-induced $1 \times 1 \rightleftharpoons 1 \times 2$ phase transition.

The measurements were performed in a standard ultrahigh-vacuum (UHV) system pumped by a combination of titanium-ion sublimation-turbomolecular pump. For measurements of partial pressures a differentially pumped mass spectrometer was attached to the chamber. Details of the experimental setup will be reported elsewhere. ${ }^{6}$ The $\mathrm{Pt}(110)$ sample, which was the same used in previous experiments, ${ }^{2-4}$ was cleaned by standard methods. Profiles of the 0,0 beam were taken with a high-resolution LEED instrument (SPALEED) of $\sim 2000 \AA$ transfer width whose operational principle has been described elsewhere. ${ }^{7}$ A kinematical analysis of the beam profiles recorded with varying electron energy allows a quantitative description of the facetted surface in terms of roughening depth and lateral terrace width distribution. 8,9

Figure 1 shows in a plot of the reaction rate $\mathrm{rCO}_{2}$ vs

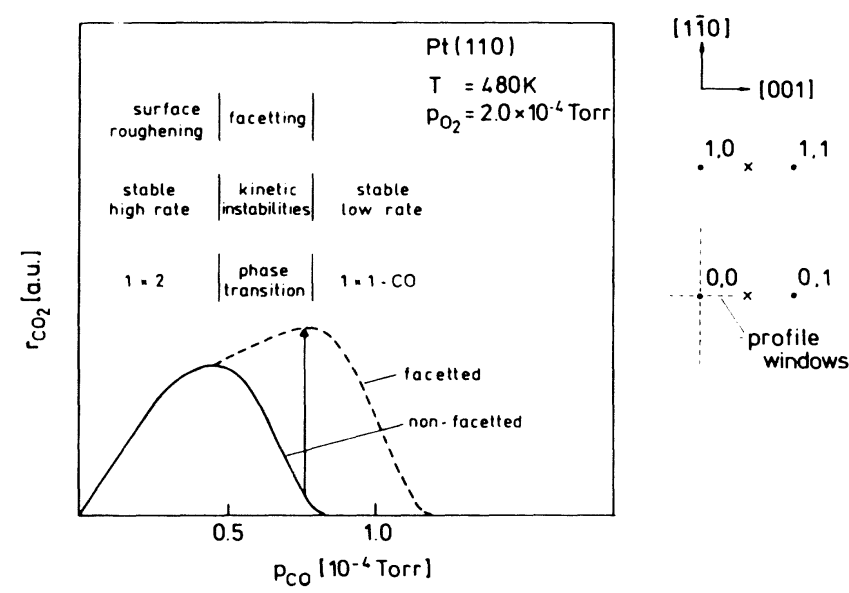

FIG. 1. Relation between the conditions for facetting and the kinetics of the catalytic $\mathrm{CO}$ oxidation on $\mathrm{Pt}(110)$. The solid line indicates the rate curve for the nonfacetted surface, while the dashed curve indicates the increase in catalytic activity after strong facetting of the surface. The different regions indicated on top of the rate curve all refer to the nonfacetted Pt(110) surface. Inset: A schematic of the LEED pattern with the $1 \times 2$ reconstruction. The dashed lines indicate the directions in which spot profiles were taken. 
$p_{\text {co }}$ the kinetics of the catalytic $\mathrm{CO}$ oxidation on $\mathrm{Pt}(110)$ exhibiting a high and low reaction-rate branch characteristic of the Langmuir-Hinshelwood (LH) mechanism. Facetting is restricted to the transition region between the two branches where the transition from an oxygen-covered $1 \times 2$ surface to a CO-covered $1 \times 1$ surface also takes place. The facetting of $\mathrm{Pt}(110)$ is associated with an increase in catalytic activity leading to the dashed rate curve in Fig. 1 whose rate maximum is shifted to higher $p_{\text {co }}$. Experimentally the facetting process at $10^{-4}$ Torr could therefore be followed in situ simply by measuring the increase of the reaction rate at constant $p_{\text {CO }}$ as indicated by the arrow in Fig. 1. Since the facets which are formed belong almost exclusively to the [001] zone, the facetting gives rise to an unidirectional broadening, respectively splitting the integralorder beams perpendicular to the direction of the $1 \times 2$ reconstruction. The experimental procedure was such that first the surface was facetted under reaction conditions at $10^{-4}$ Torr and at $T=480 \mathrm{~K}$. Beam profiles were recorded from the $\mathrm{CO}$-covered surface after rapidly cooling down the sample to $300 \mathrm{~K}$ and pumping to $p<1 \times 10^{-8}$ Torr.

After a strong facetting which led to the dashed rate curve shown in Fig. 1 the 0,0 beam exhibited a splitting into five distinct beams as demonstrated by a perspective view of the intensity distribution displayed in Fig. 2. The electron energy of $74 \mathrm{eV}$ was chosen close to inphase scattering conditions at $80 \mathrm{eV}$ for different vertical terrace levels $\left(S=K_{\perp} d / 2 \pi=2\right)$ with $K_{\perp}$ being the vertical component of the scattering vector and $d$ being the vertical layer distance of $\mathrm{Pt}(110)$. By varying the electron energy from in-phase scattering conditions at $80 \mathrm{eV}$ $(S=2)$ to out-of-phase scattering at $125 \mathrm{eV}(S=2.5)$ one obtains a series of profiles shown in Fig. 3.

From the energy dependence of the splitting of the outer satellites in Fig. 3(b), a (430) orientation of the facets could be determined. The splitting of the inner satellite spots in Fig. 3(a) shows not $K_{\perp}$ dependence close

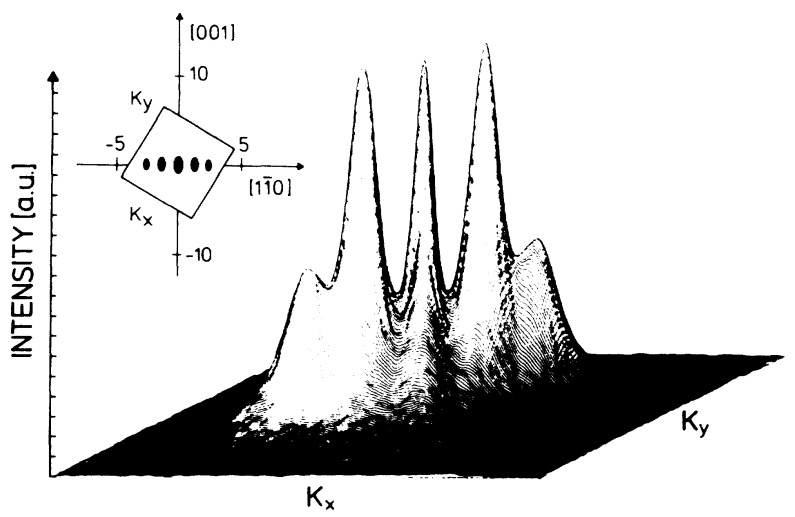

FIG. 2. Perspective view of the 0,0 -beam intensity distribution at $74 \mathrm{eV}$ after strong facetting of the surface. to in-phase scattering conditions and remains fixed at $1.35 \%$ of the distance to the next normal spot corresponding to a periodicity of 74 lattice units in real space along [1-10]. The shoulder at $S=2.12$ in Fig. 3(a) is very likely due to a third-order satellite in the diffraction pattern of the facetted surface. The appearance of higher-order satellites indicates a regular array of facets which is well ordered within the transfer width of the instrument $(\approx 2000 \AA)$. As the simplest model for the facetted surface we chose a symmetric sawtoothlike arrangement of alternating (340) and (430) facets as depicted in Fig. 4. The calculated spot intensities represented by bars in Fig. 4 show good agreement with the experimental results. The slightly higher intensity of the measured central spot can be explained by the presence of additional unfacetted (110) areas on the facetted surface.

(a)

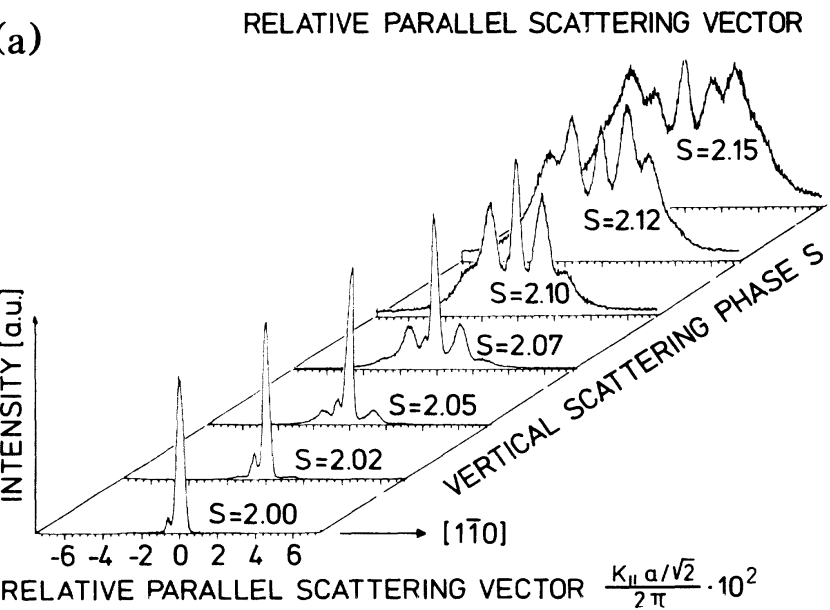

(b)

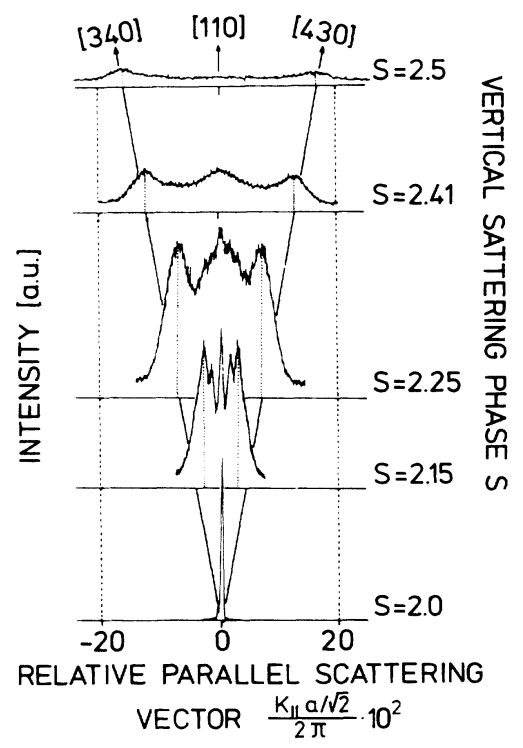

FIG. 3. Spot profiles of the 0,0 beam after strong facetting of the surface for different scattering vectors $S\left(K_{\perp} d=2 \pi S\right)$. The profiles are shown close to (a) in-phase scattering conditions and include (b) out-of-phase scattering conditions. 


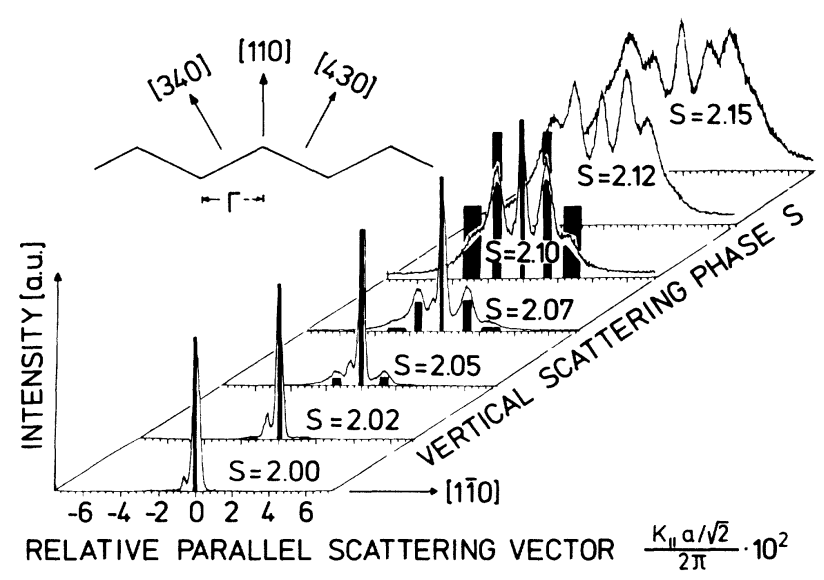

FIG. 4. Comparison between the measured spot profiles and the profiles calculated from the geometrical model of the facetted surface displayed in the inset. $2 \Gamma$ denotes the lateral periodicity of 74 lattice units along the [1 10$]$ direction found in the experiment. For better comparability the width of the columns is changed simultaneously to the width of the measured spots.

The consistency of the structural model presented above can be tested by comparing the surface roughness from the geometrical model with the roughness determined from the intensity decrease of the central 0,0 beam close to in-phase scattering conditions. The procedure which has been described in detail elsewhere ${ }^{9}$ proceeds along the following steps. The lattice factor $G\left(K_{\perp}\right)$ is obtained by a decomposition of the profile into a central peak of Gaussian shape and four satellite peaks of Lorentzian shape:

$$
G\left(K_{\perp}\right)=\frac{\int_{\mathrm{BZ}} I_{\text {central }}\left(K_{\perp}\right)}{\int_{\mathrm{BZ}} I_{\text {central }}\left(K_{\perp}\right)+\sum_{i=1}^{4} \int_{\mathrm{BZ}} I_{\text {satellite }}\left(K_{\perp}\right)} .
$$

For $K_{\perp}$ close to in-phase scattering conditions the approximation

$$
G\left(K_{\perp}\right) \simeq 1-\Delta^{2}\left(\Delta K_{\perp}\right)^{2} \simeq e^{-\Delta^{2}\left(\Delta K_{\perp}\right)^{2}}
$$

is valid. ${ }^{9}$ Here $\Delta K_{\perp}$ is the deviation of $K_{\perp}$ from the inphase scattering condition. So a plot of $\ln G(K)$ vs $\left(\Delta K_{\perp}\right)^{2}$ yields the rms roughness of the surface in the [110] direction. A value of 3.0 layers was obtained in excellent agreement with the rms roughness of 2.8 which can be extracted from the simple geometrical model displayed in Fig. 4. For comparison, an rms roughness of 0.6 had been determined for the unfacetted surface.

The dependence of the facetting process on the reaction conditions was further investigated in a number of similar experiments where $p_{\mathrm{O}_{2}}, T$, and the exposure time of $\sim 30$ min were kept constant while $p_{\text {CO }}$ was varied. Thus different regions of the LH kinetics were probed which were found to correspond essentially to the three different kinds of substrate changes indicated in Fig. 1. As reported before ${ }^{2}$ facetting could only be observed in

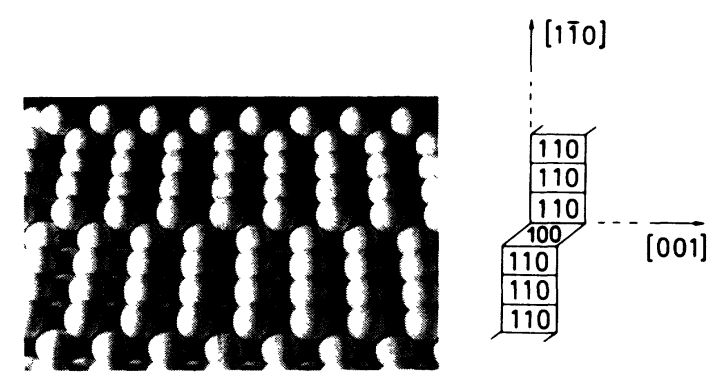

FIG. 5. Ball model of the (430) facet illustrating the structural elements of nonreconstructed (110) terrace units and (100) step units that build up the facet.

the region beyond the rate maximum up to $p_{\text {Co }}$ values where the kinetic activity of the surface had almost ceased and where also no substrate changes could be detected. But even in the stable high reaction-rate branch where no facetting occurs, the reaction caused a strong roughening of the surface which increased with $p_{\text {CO }}$ and reached an rms value of 2.6 close to the rate maximum. The position of the rate maximum therefore essentially separates ordered from disordered structural changes, while the elementary processes are apparently the same on both sides.

The elementary step in the facetting of $\operatorname{Pt}(110)$ is clearly the $1 \times 1 \rightleftharpoons 1 \times 2$ PT. This can easily be demonstrated with a ball model. The mass transport of $50 \%$ of the surface atoms associated with the PT necessarily creates steps. As demonstrated with the ball model of a (430) facet region, displayed in Fig. 5, all orientations of the [001] zone can be built up by arranging (100) steps and (110) terrace units in a regular sequence. The conditions which lead to a facetting of $\mathrm{Pt}(110)$ in $\mathrm{CO}+\mathrm{O}_{2}$ can best be rationalized if one first considers the structural changes during kinetic oscillations. These occur at higher temperature than the facetting process ( $T>530 \mathrm{~K}$ or at low temperature after completion of the facetting process). ${ }^{3,10}$ The oscillations in the rate of $\mathrm{CO}_{2}$ formation have been shown to be due to the $1 \times 1$ $\rightleftharpoons 1 \times 2$ PT which modulates the oxygen sticking coefficient and hence the catalytic activity. During kinetic oscillations no facetting occurs, since the periodic structural changes take place preferentially within one layer. Because of thermal instability of the facets, a reordering process during kinetic oscillations keeps the surface essentially flat. At lower temperature, however, thermal reordering becomes ineffective and the structural changes may then go several layers deep leading to a facetting of the surface. The necessary mass transport of $\mathrm{Pt}$ atoms is provided by the $\mathrm{PT}$ as local CO-coverage fluctuations around the critical CO coverage of the PTinduce local structural changes. The experimentally observed time and length scale is consistent with the proposed mechanism. This has been demonstrated in a computer simulation which showed that the adsorbing 
and reacting of several thousand monolayers of $\mathrm{CO}$ and $\mathrm{O}_{2}$ produces facets of similar dimensions as found in the experiment. ${ }^{11}$

The proposed mechanism describes the facetting as being due to a Turing instability which renders the flat surface unstable and causes the appearance of a periodically modulated substrate structure. A possible thermodynamical driving force for facetting is inconsistent with the experimental finding of a uniform size distribution for the facets. In this case larger facets should be favored against smaller ones. In summary, it has been shown that kinetic instabilities in a catalytic surface reaction can give rise to stationary spatial pattern formation via a facetting of the surface.

(a) Permanent address: Fritz-Haber-Institut der MaxPlanck-Gesellschaft, Faradayweg 4-6, D-1000 Berlin 33,
Federal Republic of Germany.

${ }^{1}$ M. Flytzani-Stephanopoulos and L. D. Schmidt, Prog. Surf. Sci. 9, 83 (1979); G. A. Somorjai and M. A. Van Hove, Prog. Surf. Sci. 30, 201 (1989).

${ }^{2}$ S. Ladas, R. Imbihl, and G. Ertl, Surf. Sci. 197, 153 (1988).

${ }^{3}$ S. Ladas, R. Imbihl, and G. Ertl, Surf. Sci. 198, 42 (1988).

${ }^{4}$ T. Gritsch, D. Coulman, R. J. Behm, and G. Ertl, Phys. Rev. Lett. 63, 1086 (1989).

${ }^{5}$ G. Nicolis and I. Prigogine, Self-Organization in Nonequilibrium Systems (Wiley, New York, 1977); H. Meinhardt, Models of Biological Pattern Formation (Academic, London, 1982).

${ }^{6}$ J. Falta, R. Imbihl, and M. Henzler (to be published).

${ }^{7}$ U. Scheithauer and M. Henzler, Surf. Sci. 178, 441 (1986).

${ }^{8}$ H. Busch and M. Henzler, Surf. Sci. 167, 534 (1986).

${ }^{9} \mathrm{~J}$. Wollschläger, J. Falta, and M. Henzler (to be published). ${ }^{10} \mathrm{M}$. Eiswirth and G. Ertl, Surf. Sci. 177, 90 (1986).

${ }^{11}$ R. Imbihl, A. Reynolds, and D. Kaletta (to be published). 


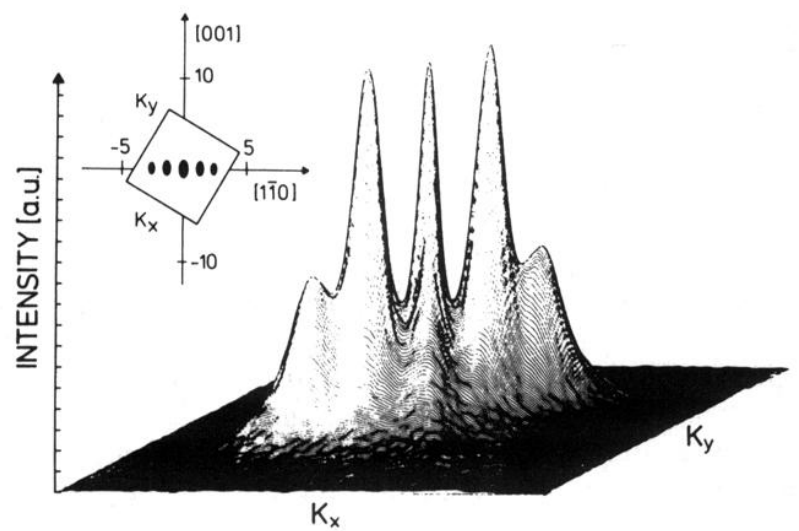

FIG. 2. Perspective view of the 0,0 -beam intensity distribution at $74 \mathrm{eV}$ after strong facetting of the surface. 


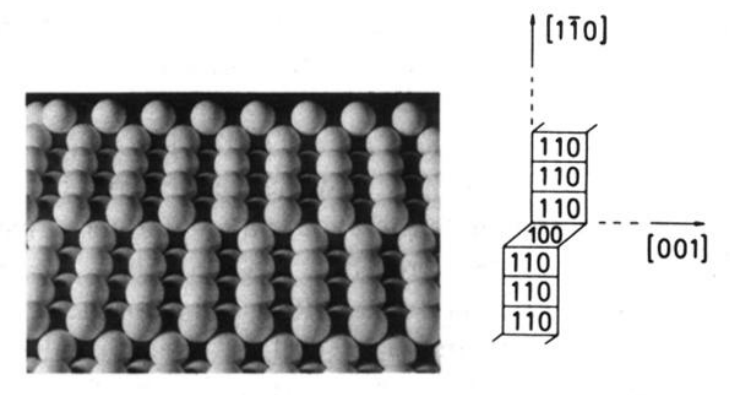

FIG. 5. Ball model of the (430) facet illustrating the structural elements of nonreconstructed (110) terrace units and (100) step units that build up the facet. 